Ergod. Th. \& Dynam. Sys. (1986), 6, 265-280

Printed in Great Britain

\title{
Topological conjugacy for sofic systems
}

\author{
MASAKAZU NASU \\ Faculty of Engineering, Mie University, Tsu 514, Japan \\ (Received 19 November 1984; revised 20 June 1985 and 23 October 1985)
}

\begin{abstract}
We prove that any topological conjugacy between subshifts is decomposed into the product of 'bipartite codes', and obtain a natural generalization of Williams' theorem to sofic systems: two sofic systems are topologically conjugate iff the 'representation matrices' of the right [left] Krieger covers for them are 'strong shift equivalent' within right [left] Krieger covers; a similar result with respect to Fischer covers holds for transitive sofic systems.
\end{abstract}

\section{Introduction}

We call a 1-block factor map of a topological Markov chain onto a sofic system a cover for the sofic system or a sofic cover (cf. [2]). It is well known that each sofic system can be defined as the image of a sofic cover. Canonical right and left resolving covers have been introduced by R. Fischer [3] for each transitive (i.e. one-sided topologically transitive) sofic system, and recently other canonical right and left resolving covers have been introduced by W. Krieger [9] for any sofic system. We call the former right and left Fischer covers and the latter right and left Krieger covers. In [9], Krieger has proved that for any topological conjugacy $\phi: \Lambda \rightarrow \Omega$ between sofic systems, if $\theta: \Sigma \rightarrow \Lambda$ and $\pi: \Gamma \rightarrow \Omega$ are the right [left] Krieger covers, then there exists a topological conjugacy $\eta: \Sigma \rightarrow \Gamma$ such that $\pi \eta=\phi \theta$. He has also proved a similar result with respect to Fischer covers for transitive sofic systems. Fischer covers have been also studied in [2], [8], and [11]. In [2], a direct proof for Krieger's result with respect to Fischer covers has been given. In this paper, we are concerned with the above canonical covers and elucidate further topological conjugacy for sofic systems.

First we introduce 'bipartite codes' and prove that any topological conjugacy between subshifts is decomposed into the product of bipartite codes. Using this result and using 'bipartite lambda graphs', we can prove results which include the above Krieger's results, and at the same time give a natural generalization of Williams' theorem [13] to sofic systems: we introduce 'representation matrices' and 'strong shift equivalence' for them and proye that two sofic systems are topologically conjugate iff the representation matrices of the right [left] Krieger covers for them are strong shift equivalent within right [left] Krieger covers; a similar result is also proved for transitive sofic systems with respect to Fischer covers. In $\S 5$, we give a practical method based on automata theory to construct the Krieger covers for a 
given sofic system. In the final section, a remark is added on topological conjugacy for sofic covers defined in [2].

\section{Bipartite codes}

Let $A$ be an alphabet (i.e. a finite set of symbols). The shift map $\sigma: A^{\mathbf{Z}} \rightarrow A^{\mathbf{Z}}$ is defined by

$$
\sigma\left(\left(\alpha_{i}\right)_{i \in \mathbf{Z}}\right)=\left(\alpha_{i+1}\right)_{i \in \mathbf{Z}}, \quad\left(\alpha_{i}\right)_{i \in \mathbf{Z}} \in A^{\mathbf{Z}} .
$$

Give $A$ the discrete topology and $A^{Z}=\prod_{-\infty}^{\infty} A$ the product topology. Let $\Sigma$ be a closed $\sigma$-invariant subset of $A^{\mathbf{Z}}$ and let $\bar{\Sigma}: \Sigma \rightarrow \Sigma$ be the restriction of $\sigma$. Then the dynamical system $(\Sigma, \bar{\Sigma})$ is called a subshift over $A$. For simplicity, $\Sigma$ will be used to denote either the subshift $(\Sigma, \bar{\Sigma})$ or its underlying space $\Sigma$. We say that a word $w \in A A^{*}$ appears on a point $\alpha=\left(\alpha_{i}\right)_{i \in \mathbf{Z}} \in \Sigma$ if $w=\alpha_{i} \alpha_{i+1} \cdots \alpha_{j}$ for some $i, j, i \leq j$, where $A^{*}$ is the free monoid generated by $A$. $L(\Sigma)$ will denote the set of all words that appear on some point of $\Sigma$ and $A(\Sigma)$ will denote the alphabet over which $\Sigma$ is defined. We assume that $A(\Sigma)$ equals the set of all symbols that appear on some point of $\Sigma$. It is well known that $L(\Sigma)$ uniquely determines $\Sigma$.

Let $\Sigma$ be a subshift and $n$ be a positive integer. Then

$$
\Sigma^{[n]}=\left\{\left(\alpha_{i} \cdots \alpha_{i+n-1}\right)_{i \in \mathbf{Z}} \mid\left(\alpha_{i}\right)_{i \in \mathbf{Z}} \in \Sigma\right\}
$$

gives a subshift $\Sigma^{[n]}$, which is called the higher block system of order $n$ of $\Sigma$ [1].

Remark 2.1. For any subshift $\Sigma$ and $n \geq 1,\left(\Sigma^{[n]}\right)^{[2]}$ is equal to $\Sigma^{[n+1]}$ up to recoding of symbols (i.e. $\Sigma^{[n+1]}$ is obtained from $\left(\Sigma^{[n]}\right)^{[2]}$ by recoding the symbols in $\left.A\left(\left(\Sigma^{[n]}\right)^{[2]}\right)\right)$.

Let $\Sigma$ and $\Omega$ be subshifts and $m$ and $n$ non-negative integers. A factor map (i.e. an onto homomorphism) $\pi: \Sigma \rightarrow \Omega$ is of $(m, n)$ type if there exists a mapping $k: A(\Sigma)^{m+n+1} \cap L(\Sigma) \rightarrow A(\Omega)$ such that

where

$$
\pi\left(\left(\alpha_{i}\right)_{i \in \mathbf{Z}}\right)=\left(\beta_{i}\right)_{i \in \mathbf{Z}}, \quad\left(\alpha_{i}\right)_{i \in \mathbf{Z}} \in \Sigma,
$$

$$
\beta_{i}=k\left(\alpha_{i-m} \alpha_{i-m+1} \cdots \alpha_{i+n}\right)
$$

for all $i \in \mathbb{Z}$. The well-known theorem of Curtis, Hedlund and Lyndon [7] asserts that any factor map is of $(m, n)$ type for some $m, n \geq 0$.

Let $A, C$ and $D$ be alphabets. A one-to-one mapping $f: A \rightarrow C D$ is called a bipartite expression of $A$.

Let $\Sigma$ and $\Omega$ be subshifts. Let $f: A(\Sigma) \rightarrow C D$ be a bipartite expression of $A(\Sigma)$. A mapping $\phi: \Sigma \rightarrow \Omega$ is called a bipartite code induced by $f$ if there exists a bipartite expression $\tilde{f}: A(\Omega) \rightarrow D C$ such that either of the following (i) or (ii) is the case:

(i) If $\alpha=\left(\alpha_{i}\right)_{i \in \mathbf{Z}} \in \Sigma, \phi(\alpha)=\left(\beta_{i}\right)_{i \in \mathbb{Z}}$ and $f\left(\alpha_{i}\right)=c_{i} d_{i}$ with $c_{i} \in C$ and $d_{i} \in D$ for $i \in \mathbb{Z}$, then $\tilde{f}\left(\beta_{i}\right)=d_{i} c_{i+1}$ for all $i \in \mathbb{Z}$.

(ii) With the same assumption as in (i), $\tilde{f}\left(\beta_{i}\right)=d_{i-1} c_{i}$ for all $i \in \mathbb{Z}$.

Particularly, the mapping $\phi: \Sigma \rightarrow \Omega$ such that with the same assumption as in (i), $\beta_{i}=d_{i} c_{i+1}$ for all $i \in \mathbb{Z}$, will be called the standard bipartite code induced by $f . \mathrm{A}$ mapping $\phi: \Sigma \rightarrow \Omega$ is called a bipartite code if $\phi$ is a bipartite code induced by some bipartite expression of $A(\Sigma)$. 
It is clear that a bipartite code is a topological conjugacy and if $\phi$ is a bipartite code, then so is $\phi^{-1}$.

Remark 2.2 For a subshift $\Sigma$ with $A(\Sigma)=A$, the bipartite expression $f: A \rightarrow A\{1\}$ defined by $f(a)=a 1 \quad a \in A$, induces the shift map $\bar{\Sigma}$ on $\Sigma$.

Remark 2.3. Let $\Sigma$ be a subshift and $\Sigma^{[2]}$ the higher block system of order 2 of $\Sigma$. The natural conjugacy $\rho$ from $\Sigma$ to $\Sigma^{[2]}$, i.e. the conjugacy $\rho: \Sigma \rightarrow \Sigma^{[2]}$ defined by

$$
\rho\left(\left(\alpha_{i}\right)_{i \in \mathbf{Z}}\right)=\left(\left(\alpha_{i} \boldsymbol{\alpha}_{i+1}\right)\right)_{i \in \mathbf{Z}}, \quad\left(\left(\alpha_{i}\right)_{i \in \mathbf{Z}} \in \mathbf{\Sigma}\right)
$$

is a bipartite code induced by the bipartite expression $f: A \rightarrow A A$ defined by $f(a)=a a$ $(a \in A)$.

THEOREM 2.4. Any topological conjugacy between subshifts is decomposed into the product of bipartite codes.

To prove the theorem, first we prove:

LEMMA 2.5. Let $\phi: \Delta \rightarrow \Omega$ be a conjugacy of $(0,0)$ type between subshifts and let it be given by $h: A(\Delta) \rightarrow A(\Omega)$. Let $\phi^{-1}$ be of $(p, q)$ type with $p, q \geq 0$. Let $f$ be the bipartite expression of $A(\Delta)$ defined by

$$
\begin{aligned}
f(a) & =h(a) a, & & a \in A(\Delta), \\
{[f(a)} & =a h(a), & & a \in A(\Delta)] .
\end{aligned}
$$

Let $\psi_{f}$ be the standard bipartite code induced by $f$. Let $\Gamma=\psi_{f}(\Delta)$. Let $\Omega^{[2]}$ be the higher block system of order 2 of $\Omega$ and let $\rho$ be the natural conjugacy from $\Omega$ to $\Omega^{[2]}$. Then there exists a conjugacy $\Phi: \Gamma \rightarrow \Omega^{[2]}$ of $(0,0)$ type such that $\Phi^{-1}$ is of $(p, q-1)$ type $[(p-1, q)$ type $]$ and the diagram

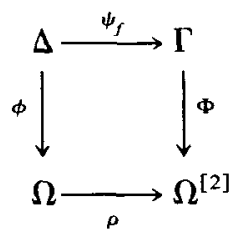

commutes.

Proof. Since $\phi$ is given by $h$, we have

$$
\phi\left(\left(\alpha_{i}\right)_{i \in \mathbf{Z}}\right)=\left(h\left(\alpha_{i}\right)\right)_{i \in \mathbf{Z}}, \quad\left(\alpha_{i}\right)_{i \in \mathbf{Z}} \in \Delta .
$$

Since $\phi^{-1}$ is of $(p, q)$ type, there exists a mapping $k: A(\Omega)^{p+q+1} \cap L(\Omega) \rightarrow A(\Delta)$ such that

$$
k\left(h\left(a_{1}\right) \cdots h\left(a_{p+q+1}\right)\right)=a_{p+1}
$$

for $a_{1} \cdots a_{p+q+1} \in L(\Delta)$ with $a_{i} \in A(\Delta)$. Since $\psi_{f}$ is the standard bipartite code induced by $f, \Gamma$ is given by

$$
\Gamma=\left\{\left(\alpha_{i} h\left(\alpha_{i+1}\right)\right)_{i \in \mathbf{Z}} \mid\left(\alpha_{i}\right)_{i \in \mathbf{Z}} \in \Delta\right\} .
$$

We define a mapping $H: A(\Gamma) \rightarrow A\left(\Omega^{[2]}\right)$ by

$$
H\left(a_{1} h\left(a_{2}\right)\right)=h\left(a_{1}\right) h\left(a_{2}\right),
$$


where $a_{1} a_{2} \in L(\Delta)$ with $a_{1}, a_{2} \in A(\Delta)$. Let $\Phi: \Gamma \rightarrow \Omega^{[2]}$ be the factor map defined by

$$
\Phi\left(\left(\gamma_{i}\right)_{i \in \mathbf{Z}}\right)=\left(H\left(\gamma_{i}\right)\right)_{i \in \mathbf{Z}}, \quad\left(\gamma_{i}\right)_{i \in \mathbf{Z}} \in \Gamma \text {. }
$$

It is clear that $\Phi \psi_{f}=\rho \phi$ and $\Phi$ is a conjugacy.

Considering $k$, we can define a mapping $K: A\left(\Omega^{[2]}\right)^{p+q} \cap L\left(\Omega^{[2]}\right) \rightarrow A(\Gamma)$ by

$$
K\left(\left(h\left(a_{1}\right) h\left(a_{2}\right)\right)\left(h\left(a_{2}\right) h\left(a_{3}\right)\right) \cdots\left(h\left(a_{p+q}\right) h\left(a_{p+q+1}\right)\right)\right)=a_{p+1} h\left(a_{p+2}\right),
$$

where $a_{1} \cdots a_{p+q+1} \in L(\Delta)$ with $a_{i} \in A(\Delta)$. Since

$$
K\left(H\left(c_{1}\right) \cdots H\left(c_{p+q}\right)\right)=c_{p+1}
$$

for $c_{1} \cdots c_{p+q} \in L(\Gamma)$ with $c_{i} \in A(\Gamma), \Phi^{-1}$ is given by $K$ and is of $(p, q-1)$ type.

Thus we have proved the first version of the lemma; the proof of the second version is similar.

Let $\pi: \Lambda \rightarrow \Omega$ be any conjugacy between subshifts. Let $\pi$ be of $(m, n)$ type with $m, n \geq 0$. Let $\Lambda^{(i)}, i=0, \ldots, m+n$, be such that $\Lambda^{(0)}=\Lambda$ and $\Lambda^{(i)}$ is the higher block system of order 2 of $\Lambda^{(i-1)}$ for $i=1, \ldots, m+n$. Let $\xi^{(i)}: \Lambda^{(i-1)} \rightarrow \Lambda^{(i)}$ be the natural conjugacy for $i=1, \ldots, m+n$. Then there exists a conjugacy $\phi: \Lambda^{(m+n)} \rightarrow \Omega$ of $(0,0)$ type such that

$$
\pi=\phi \xi^{(m+n)} \xi^{(m+n-1)} \cdots \xi^{(1)}(\bar{\Lambda})^{-m}
$$

where $\bar{\Lambda}$ is the shift map on $\Lambda$.

Put $\Delta=\Lambda^{(m+n)}$. Let $\phi$ be given by $h: A(\Delta) \rightarrow A(\Omega)$. Let $\phi^{-1}$ be of $(p, q)$ type with $p, q \geq 0$. Let $\Omega^{(i)}, i=0, \ldots, p+q$, be such that $\Omega^{(0)}=\Omega$ and $\Omega^{(i)}$ is the higher block system of order 2 of $\Omega^{(i-1)}$ for $i=1, \ldots, p+q$. Let $\rho^{(i)}: \Omega^{(i-1)} \rightarrow \Omega^{(i)}$ be the natural conjugacy for $i=1, \ldots, p+q$. Then using the first version of lemma $2.5 q$ times and then using the second version of it $p$ times, we obtain the subshifts $\Delta_{i}, i=0, \ldots, p+q$, with $\Delta_{0}=\Delta$, and bipartite codes $\psi_{i}: \Delta_{i-1} \rightarrow \Delta_{i}, i=1, \ldots, p+q$, conjugacies $\phi_{i}: \Delta_{i} \rightarrow \Omega^{(i)}$ of $(0,0)$ type, $i=1, \ldots, p+q$, such that the diagram

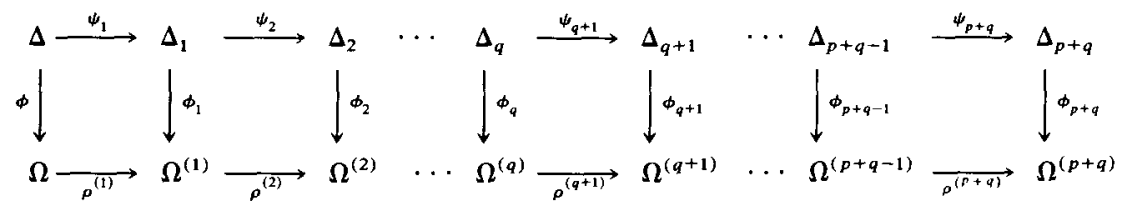

commutes and $\phi_{i}^{-1}$ is of $(p, q-i)$ type for $i=1, \ldots, q$ and of $(p+q-i, 0)$ type for $i=q+1, \ldots, p+q$.

By (2.1) and (2.2) we have

$$
\pi=\left(\rho^{(1)}\right)^{-1} \cdots\left(\rho^{(p+q)}\right)^{-1} \phi_{p+q} \psi_{p+q} \cdots \psi_{1} \xi^{(m+n)} \cdots \xi^{(1)}(\bar{\Lambda})^{-m}
$$

Since both of $\phi_{p+q}$ and $\phi_{p+q}^{-1}$ are of $(0,0)$ type, $\phi_{p+q}$ is given by a one-to-one mapping of $A\left(\Delta_{p+q}\right)$ onto $A\left(\Omega^{(p+q)}\right)$. Therefore $\phi_{p+q} \psi_{p+q}$ is a bipartite code. Thus, by remarks 2.2 and 2.3 and the fact that the inverse of a bipartite code is also a bipartite code, theorem 2.4 is proved.

\section{Strong shift equivalence of canonical sofic covers}

A (directed) graph $G$ consists of a finite set $U$ of vertices, called the vertex-set of $G$, a finite set $R$ of (directed) arcs, called the arc-set of $G$, and two mappings $i_{G}: R \rightarrow U$ and $t_{G}: R \rightarrow U$. (If $G$ is understood, we will often use $i$ and $t$ instead of 
$i_{G}$ and $t_{G}$.) For each arc $r \in R, i(r)$ and $t(r)$ are called the initial vertex of $r$ and the terminal vertex of $r$, respectively. We also say that $r$ goes from $u$ to $v$ for $u, v \in U$, if $u=i(r)$ and $v=t(r)$. It is convenient to regard each arc as a symbol and the arc-set $R$ as an alphabet. Hence, $A(G)$ will denote $R$ and $L(G)$ will denote the set of all words $r_{1} \cdots r_{k}, k \geq 1, r_{i} \in R$ such that $t\left(r_{i-1}\right)=i\left(r_{i}\right)$ for $i=1, \ldots, k$. If $x \in R^{k} \cap$ $L(G)$ for $k \geq 1$, then $x$ is called a path of length $k$ in $G$. We extend $i_{G}$ and $t_{G}$ to mappings of $L(G)$ into $U$ as follows: if $x=r_{1} \cdots r_{k} \in L(G)$ with $r_{i} \in R$, then $i_{G}(x)=i_{G}\left(r_{1}\right)$ and $t_{G}(x)=t_{G}\left(r_{k}\right)$. For each $x \in L(G)$, we call $i(x)$ the initial vertex of $x$ and $t(x)$ the terminal vertex of $x$, and say that $x$ goes from $u$ to $v$ for $u, v \in U$ if $i(x)=u$ and $t(x)=v$.

A graph $G$ is said to be non-degenerate if both of $i_{G}$ and $t_{G}$ are onto, and irreducible if for any vertices $u$ and $v$, there exists $x \in L(G)$ such that $x$ goes from $u$ to $v$.

A subshift $\Sigma$ is called a topological Markov chain if there exists a non-degenerate graph $G$ such that $L(\Sigma)=L(G)$, and a sofic system if it is the image of a factor map from some topological Markov chain [12]. It is well known that a subshift is a sofic system iff it is the image of a factor map of $(0,0)$ type from some topological Markov chain. A factor map of $(0,0)$ type from a topological Markov chain to a sofic system, is called a cover for the sofic system or a sofic cover (cf. [2]).

Each sofic cover $\phi: \Sigma \rightarrow \Omega$ is naturally represented by a non-degenerate graph $G$ with $L(G)=L(\Sigma)$ such that each arc $r$ of $G$ is labelled $h(r)$ where $h$ is the mapping of $A(\Sigma)$ onto $A(\Omega)$ that gives $\phi$. Such a labelled graph will generally be called a lambda graph. A similar notion is well known in automata theory as the transition diagram of a (non-deterministic) finite automaton (see e.g. [6]). Formally, a lambda graph ( $\lambda$-graph) $\mathscr{G}$ over an alphabet $A$ is given by $\mathscr{G}=(G, \lambda)$ where $G$ is a graph and $\lambda$ is a mapping of $A(G)$ onto $A$. We naturally extend $\lambda$ to a mapping of $L(G)$ into $A A^{*}$, that is, for each $x=r_{1} \cdots r_{k} \in L(G)$ with $r_{i} \in A(G)$, we define $\lambda(x)$ to be $\lambda\left(r_{1}\right) \cdots \lambda\left(r_{k}\right)$, which will be called the word generated by the path $x$. Moreover, let $A(\mathscr{G})$ denote $A$ and define

$$
L(\mathscr{G})=\{\lambda(x) \mid x \in L(G)\},
$$

which will be called the language generated by $\mathscr{G}$. For each vertex $u$ of $\mathscr{G}$ (i.e. of $G)$, we define

$$
L_{u}(\mathscr{G})=\{\lambda(x) \mid x \in L(G), i(x)=u\}
$$

We say that $\mathscr{G}$ is non-degenerate or irreducible according to whether $G$ is nondegenerate or irreducible, respectively. $\mathscr{G}$ is said to be right [left] resolving if for any distinct two arcs $r$ and $s$ of $\mathscr{G}$ with $i(r)=i(s)[t(r)=t(s)], \lambda(r)$ and $\lambda(s)$ are different. For a right resolving $\lambda$-graph $\mathscr{G}$, two vertices $u$ and $v$ of $\mathscr{G}$ are said to be equivalent if $L_{u}(\mathscr{G})=L_{v}(\mathscr{G})$. We say that a right resolving $\lambda$-graph $\mathscr{G}$ is reduced if it has no distinct equivalent vertices.

Two $\lambda$-graphs $\mathscr{G}_{1}=\left(G_{1}, \lambda_{1}\right)$ and $\mathscr{G}_{2}=\left(G_{2}, \lambda_{2}\right)$ are said to be isomorphic if there exists a pair of bijections $g: U_{1} \rightarrow U_{2}$ and $h: A\left(G_{1}\right) \rightarrow A\left(G_{2}\right)$ such that $g i_{G_{1}}=i_{G_{2}} h$, $g t_{G_{1}}=t_{G_{2}} h$ and $\lambda_{1}=\lambda_{2} h$, where $U_{i}$ is the vertex-set of $G_{i}$ for $i=1,2$. The pair $(g, h)$ is called an isomorphism of $\mathscr{G}_{1}$ onto $\mathscr{G}_{2}$. In what follows, isomorphic $\lambda$-graphs will be identified. 
For a $\lambda$-graph $\mathscr{G}=(G, \lambda)$, a $\lambda$-graph $\mathscr{G}^{\prime}=\left(G^{\prime}, \lambda^{\prime}\right)$ is called a $s u b$ - $\lambda$-graph of $\mathscr{G}$ if $A\left(G^{\prime}\right) \subset A(G)$, the vertex-set of $G^{\prime}$ is $i_{G}\left(A\left(G^{\prime}\right)\right) \cup t_{G}\left(A\left(G^{\prime}\right)\right), i_{G^{\prime}}=i_{G} \mid A\left(G^{\prime}\right), t_{G^{\prime}}=$ $t_{G} \mid A\left(G^{\prime}\right)$ and $\lambda^{\prime}=\lambda \mid A\left(G^{\prime}\right)$.

Let $A$ be an alphabet. We call a sequence

$$
\cdots \gamma_{-1} \gamma_{0}
$$

with $\gamma_{-i} \in A, i \geq 0$, a left infinite sequence over $A$. For $\alpha=\left(\alpha_{i}\right)_{i \in \mathbf{Z}} \in A^{\mathbf{Z}}$, let $\alpha_{-}$denote the left infinite sequence $\cdots \alpha_{-1} \alpha_{0}$. For a left infinite sequence $\gamma=\cdots \gamma_{-1} \gamma_{0}$ over $A$ and $x \in A A^{*}, \gamma x$ denotes the left infinite sequence $\delta=\cdots \delta_{-1} \delta_{0}$ such that if $x$ is of length $i$ then $\delta_{1-i} \delta_{2-i} \cdots \delta_{0}=x$ and $\delta_{-j-i}=\gamma_{-j}$ for all $j \geq 0$.

Let $\Lambda$ be a subshift. Let

$$
\Lambda_{-}=\left\{\alpha_{-} \mid \alpha \in \Lambda\right\}
$$

We define an equivalence relation $E_{\Lambda}$ on $\Lambda_{-}$as follows: for $\gamma, \delta \in \Lambda_{-}, \gamma E_{\Lambda} \delta$ if

$$
\left\{x \in A A^{*} \mid \gamma x \in \Lambda_{-}\right\}=\left\{x \in A A^{*} \mid \delta x \in \Lambda_{-}\right\} .
$$

It is clear that if $\gamma E_{\Lambda} \delta$, then $\gamma x E_{\Lambda} \delta x$ for all $x \in A A^{*}$ such that $\gamma x \in \Lambda_{-}$. The equivalence class containing $\gamma \in \Lambda_{-}$will be denoted by $E_{\Lambda}[\gamma]$. If $\Lambda$ is a sofic system, then

$$
\left\{E_{\Lambda}[\gamma] \mid \gamma \in \Lambda_{-}\right\}
$$

is finite [9]. For a sofic system $\Lambda$, we define a $\lambda$-graph $\mathscr{K}_{\Lambda}$ as follows: the vertex-set of $\mathscr{K}_{\Lambda}$ is $\left\{E_{\Lambda}[\gamma] \mid \gamma \in \Lambda_{-}\right\}$and for each vertex $E_{\Lambda}[\gamma], \gamma \in \Lambda_{-}$, and $a \in A$, a unique arc labelled $a$ goes from $E_{\Lambda}[\gamma]$ to $E_{\Lambda}[\gamma a]$ if $\gamma a \in \Lambda_{-}$. The $\lambda$-graph $\mathscr{K}_{\Lambda}$ will be called the Krieger $\lambda$-graph for $\Lambda$. Clearly $\mathscr{K}_{\Lambda}$ generates $L(\Lambda)$ and it is non-degenerate, right resolving and reduced. The sofic cover represented by $\mathscr{K}_{\Lambda}$ is called the right Krieger cover for $\Lambda$, which is the same as the 'future cover' for $\Lambda$ introduced by Krieger [9].

The left Krieger cover for $\Lambda$, which is the same as the 'past cover' for $\Lambda$ in [9], is defined to be the cover represented by the $\lambda$-graph $\left(\mathscr{K}_{\Lambda^{t}}\right)^{t}$ obtained from $\mathscr{K}_{\Lambda^{t}}$ by reversing the direction of the arcs, where $\Lambda^{t}$ denotes the sofic system obtained from $\Lambda$ by reversing the direction of time.

A $\lambda$-graph $\mathscr{G}$ is called a Krieger $\lambda$-graph if $\mathscr{G}$ is equal to $\mathscr{K}_{\Lambda}$ for the sofic system $\Lambda$ such that $L(\Lambda)=L(\mathscr{G})$.

An irreducible $\lambda$-graph $\mathscr{F}$ is called a Fischer $\lambda$-graph, if $\mathscr{F}$ is right-resolving and reduced. If a subshift $\Lambda$ has the property that for any $x, y \in L(\Lambda)$ there exists $z \in L(\Lambda)$ with $x z y \in L(\Lambda)$, then $\Lambda$ is said to be transitive. As is well known, a subshift $\Lambda$ is transitive iff it is one-sided topologically transitive, i.e. there exists a point $\alpha \in \Lambda$ such that $\left\{\bar{\Lambda}^{n} \alpha \mid n \geq 0\right\}$ is dense, where $\bar{\Lambda}$ is the shift map on $\Lambda$. For any transitive sofic system $\Lambda$, there exists a unique Fischer $\lambda$-graph $\mathscr{F}$ such that $L(\mathscr{F})=L(\Lambda)$ and this equals the right resolving $\lambda$-graph with the smallest number of vertices that generates $L(\Lambda)$ [3]. This Fischer $\lambda$-graph will be called the Fischer $\lambda$-graph for $\Lambda$ and is denoted by $\mathscr{F}_{\Lambda} . \mathscr{F}_{\Lambda}$ is the unique 'ergodic component' of $\mathscr{K}_{\Lambda}$ [9]. (An ergodic component of a $\lambda$-graph $\mathscr{G}$ means a maximal irreducible sub- $\lambda$-graph $\tilde{G}$ of $\mathscr{G}$ such that no path in $\mathscr{G}$ goes from a vertex in $\tilde{\mathscr{G}}$ to the outside of $\tilde{\mathscr{G}}_{\text {.) }}$ The sofic cover represented by $\mathscr{F}_{\Lambda}$ is called the right Fischer cover for $\Lambda$. The left Fischer cover for $\Lambda$ is defined to be the cover represented by $\left(\mathscr{F}_{\Lambda^{t}}\right)^{t}$. 
The following two theorems have been proved by Krieger [9] (the second theorem has been recovered with a direct proof in [2]).

THEOREM 3.1 [Krieger]. Let $\phi: \Lambda \rightarrow \Omega$ be a topological conjugacy between sofic systems. Let $\theta: \Sigma \rightarrow \Lambda$ and $\pi: \Gamma \rightarrow \Omega$ be the right [left] Krieger covers for $\Lambda$ and $\Omega$, respectively. Then there exists a topological conjugacy $\eta: \Sigma \rightarrow \Gamma$ such that the diagram

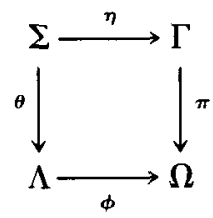

commutes.

THEOREM 3.2 [Krieger]. Let $\phi: \Lambda \rightarrow \Omega$ be a topological conjugacy between transitive sofic systems. Let $\theta: \Sigma \rightarrow \Lambda$ and $\pi: \Gamma \rightarrow \Omega$ be the right [left] Fischer covers for $\Lambda$ and $\Omega$, respectively. Then there exists a topological conjugacy $\eta: \Sigma \rightarrow \Gamma$ such that the diagram (3.1) commutes.

Krieger has also proved the uniqueness of the $\eta$ in theorems 3.1 and 3.2, in [10] and [9].

The following two theorems include the above Krieger's theorems in conjunction with theorem 2.4 .

TheOREM 3.3. Let $\phi: \Lambda \rightarrow \Omega$ be a topological conjugacy between sofic systems. Let $\phi=\psi_{1} \cdots \psi_{n}$ be a decomposition of $\phi$ into the product of bipartite codes $\psi_{i}: \Lambda_{i-1} \rightarrow \Lambda_{i}$, $i=1, \ldots, n$, with $\Lambda_{0}=\Lambda$ and $\Lambda_{n}=\Omega$. Let $\theta_{i}: \Sigma_{i} \rightarrow \Lambda_{i}$ be the right [left] Krieger cover for $\Lambda_{i}$ for $i=0, \ldots, n$. Then there exist bipartite codes $\eta_{i}: \Sigma_{i-1} \rightarrow \Sigma_{i}, i=1, \ldots, n$, such that the diagram

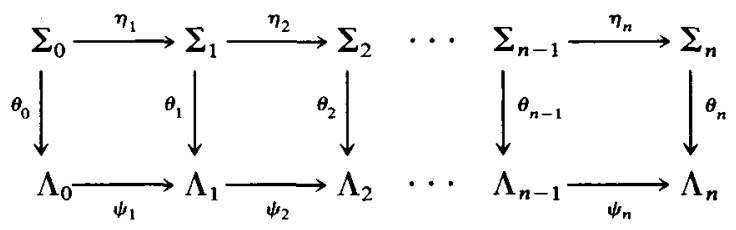

commutes.

THEOREM 3.4. Let $\phi: \Lambda \rightarrow \Omega$ be a topological conjugacy between transitive sofic systems. Let $\phi=\psi_{1} \cdots \psi_{n}$ be a decomposition of $\phi$ into the product of bipartite codes $\psi_{i}: \Lambda_{i-1} \rightarrow \Lambda_{i}, i=1, \ldots, n$, with $\Lambda_{0}=\Lambda$ and $\Lambda_{n}=\Omega$. Let $\theta_{i}: \Sigma_{i} \rightarrow \Lambda_{i}$ be the right [left] Fischer cover for $\Lambda_{i}$ for $i=0, \ldots, n$. Then there exist bipartite codes $\eta_{i}: \Sigma_{i-1} \rightarrow \Sigma_{i}$, $i=1, \ldots, n$, such that the diagram (3.2) commutes.

To state a generalization of Williams' theorem [13] to sofic systems, we need to introduce the notion of representation matrices.

For an alphabet $A$, a representation matrix over $A$ is defined to be a rectangular matrix having formal sums of the form

$$
\sum_{a \in A} k(a) a
$$


as its entries, where $k(a)$ is a non-negative integral coefficient of $a$. For alphabets $C$ and $D$, a representation matrix over $C D$ is similarly defined. For example,

$$
\left(\begin{array}{ccc}
a & b+c & 0 \\
0 & 2 b & a \\
a & b & c
\end{array}\right)
$$

is a representation matrix over $\{a, b, c\}$, and

$$
\left(\begin{array}{cc}
a a & a b+b a \\
2 b a & 0
\end{array}\right)
$$

is a representation matrix over $C D$ with $C=\{a, b\}$ and $D=\{a, b\}$.

Each $\lambda$-graph over an alphabet $A$ is described by a square representation matrix over $A$. In fact, for a $\lambda$-graph $\mathscr{G}=(G, \lambda)$ over $A$ with vertex-set $U$, a representation matrix $M(\mathscr{G})$ over $A$ indexed by $U \times U$ is defined by $M(\mathscr{G})=\left(m_{u v}\right)$ where

$$
m_{u v}=\sum \lambda(r), \quad u, v \in U,
$$

where the summation is taken over all arcs $r \in A(G)$ such that $i_{G}(r)=u$ and $t_{G}(r)=v$. $M(\mathscr{G})$ is called the representation matrix of $\mathscr{G}$. Clearly we have the unique $\lambda$-graph $\mathscr{G}$ over $\boldsymbol{A}$ with $M(\mathscr{G})=M$ for each square representation matrix $M$ over $A$. For a sofic cover $\theta, M(\mathscr{G})$ is called the representation matrix of $\theta$, when $\mathscr{G}$ is the $\lambda$-graph representing $\theta$.

If $M$ is an $m \times n$ representation matrix over an alphabet $C$ and $N$ is an $n \times m$ representation matrix over an alphabet $D$, then the (formal) product $M N$ is an $m \times m$ representation matrix over $C D$. For example,

$$
\left(\begin{array}{cc}
a & b \\
0 & a \\
b & 0
\end{array}\right)\left(\begin{array}{ccc}
a & 0 & 0 \\
0 & a & b
\end{array}\right)=\left(\begin{array}{ccc}
a a & b a & b b \\
0 & a a & a b \\
b a & 0 & 0
\end{array}\right)
$$

Two representation matrices $M$ and $N$ are said to be equal mod bijection of words if there exists a bijection of the set of all words that appear in some non-zero entry of $M$ onto the set of all words that appear in some non-zero entry of $N$ and if $M$ and $N$ are the same if we identify every pair of words connected by that bijection (note that a symbol is a word of length 1). We write $M \simeq N$ to mean that $M$ and $N$ are equal mod bijection of words. For example,

$$
\left(\begin{array}{cc}
a & b+c \\
2 b & 0
\end{array}\right) \simeq\left(\begin{array}{cc}
a a & a b+b a \\
2 b a & 0
\end{array}\right),
$$

because the mapping $h:\{a, b, c\} \rightarrow\{a a, a b, b a\}$ defined by $h(a)=a a, h(b)=b a$ and $h(c)=a b$ is a bijection. Also we have

$$
\left(\begin{array}{cc}
a a & a b+b a \\
2 b a & 0
\end{array}\right) \simeq\left(\begin{array}{cc}
a c & a d+d d \\
2 a d & 0
\end{array}\right) \simeq\left(\begin{array}{cc}
b & d+e \\
2 e & 0
\end{array}\right) .
$$

Two square representation matrices $M$ and $N$ are said to be strong shift equivalent (in l steps) if there exist alphabets $C_{i}$ and $D_{i}, i=1, \ldots, l$, and rectangular representation matrices $P_{i}$ over $C_{i}$ and $Q_{i}$ over $D_{i}, i=1, \ldots, l$, such that

$$
M \approx P_{1} Q_{1}, \quad Q_{1} P_{1} \simeq P_{2} Q_{2}, \quad \cdots, \quad Q_{l-1} P_{l-1} \simeq P_{l} Q_{l}, \quad Q_{l} P_{l} \simeq N .
$$


In particular, if $M, N$, and $Q_{i} P_{i}, i=1, \ldots, l-1$, are equal to the representation matrices of right [left] Krieger covers mod bijection of words in the above, we say that $M$ and $N$ are strong shift equivalent within right [left] Krieger covers. Strong shift equivalence within right [left] Fischer covers is similarly defined.

The following theorems are natural generalizations of Williams' theorem [13] characterizing topological conjugacy for topological Markov chains. We note that for a topological Markov chain, its defining non-negative integral matrix (see [13]) represents both the right and left Krieger cover for it.

THEOREM 3.5. Two sofic systems are topologically conjugate iff the representation matrices of the right [left] Krieger covers for them are strong shift equivalent within right [left] Krieger covers.

THEOREM 3.6. Two transitive sofic systems are topologically conjugate iff the representation matrices of the right [left] Fischer covers for them are strong shift equivalent within right [left] Fischer covers.

As will be seen in the proofs of the above theorems, the 'if' parts of the statements of them can be strengthened greatly (see also $\S 6$ ).

Proofs of theorems 3.3-3.6 will be given in the next section.

\section{Bipartite $\lambda$-graphs}

A $\lambda$-graph $\mathscr{G}=(G, \lambda)$ is said to be bipartite if $G$ is bipartite, that is, the vertex-set of $G$ can be partitioned into two sets $U_{1}$ and $U_{2}$ such that there exists no arc whose initial and terminal vertices belong to the same set. The sets $U_{1}$ and $U_{2}$ are called the bipartite vertex-sets of the bipartite graph $G$ or the bipartite $\lambda$-graph $\mathscr{G}$. (The bipartite vertex-sets are not always unique for a bipartite graph, but when we mention a bipartite graph or a bipartite $\lambda$-graph, we assume that the bipartite vertex-sets are specified.)

Let $\mathscr{B}=(G, \lambda)$ be a bipartite $\lambda$-graph with bipartite vertex-sets $U_{1}$ and $U_{2}$. For $i=1,2$, let $W_{i}$ be the set of all paths $w$ of length 2 with $i_{G}(w), t_{G}(w) \in U_{i}$. Let $A_{i}=\lambda\left(W_{i}\right)$ for $i=1,2$. We define two $\lambda$-graphs $\mathscr{G}_{1}=\left(G_{1}, \lambda_{1}\right)$ over $A_{1}$ and $\mathscr{G}_{2}=$ $\left(G_{2}, \lambda_{2}\right)$ over $A_{2}$ as follows: for $i=1,2, G_{i}$ has vertex-set $U_{i}$ and arc-set $W_{i}$; for each $w \in W_{i}, i_{G_{i}}(w)=i_{G}(w), t_{G_{i}}(w)=t_{G}(w)$ and $\lambda_{i}(w)=\lambda(w)$. We call $\mathscr{G}_{1}$ and $\mathscr{G}_{2}$ the induced pair of $\lambda$-graphs of $\mathscr{B}$. Let

$$
\begin{aligned}
& R=\left\{r \in A(G) \mid i_{G}(r) \in U_{1}, t_{G}(r) \in U_{2}\right\}, \\
& S=\left\{r \in A(G) \mid i_{G}(r) \in U_{2}, t_{G}(r) \in U_{1}\right\},
\end{aligned}
$$

and let

$$
C=\lambda(R) \text { and } \quad D=\lambda(S)
$$

Remark 4.1. We can write

$$
M(\mathscr{B})=\left(\begin{array}{ll}
0 & P \\
Q & 0
\end{array}\right),
$$

where $P$ is a representation matrix over $C$ indexed by $U_{1} \times U_{2}$ and $Q$ is that over 
$D$ indexed by $U_{2} \times U_{1}$. Clearly

$$
M(\mathscr{B})^{2}=\left(\begin{array}{cc}
M\left(\mathscr{G}_{1}\right) & 0 \\
0 & M\left(\mathscr{G}_{2}\right)
\end{array}\right),
$$

and hence $M\left(\mathscr{G}_{1}\right)=P Q$ and $M\left(\mathscr{G}_{2}\right)=Q P$.

Remark 4.2. Let $\mathscr{B}$ be non-degenerate. Let $\theta_{i}: \Sigma_{i} \rightarrow \Omega_{i}$ be the sofic cover represented by $\mathscr{G}_{i}\left(\Sigma_{i}\right.$ is the topological Markov chain such that $L\left(\Sigma_{i}\right)=L\left(G_{i}\right)$ and $\Omega_{i}$ is the sofic system such that $L\left(\Omega_{i}\right)=L\left(\mathscr{G}_{i}\right)$ ) for $i=1$, 2. Let $f: A\left(G_{1}\right) \rightarrow R S$ and $g: A_{1} \rightarrow C D$ be the embeddings. Let $\eta: \Sigma_{1} \rightarrow \Sigma_{2}$ and $\phi: \Omega_{1} \rightarrow \Omega_{2}$ be the standard bipartite codes induced by $f$ and $g$, respectively. Then the diagram

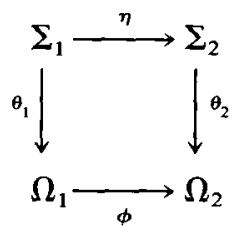

commutes.

Remark 4.3. Both of $\mathscr{G}_{1}$ and $\mathscr{G}_{2}$ are non-degenerate [irreducible] iff $\mathscr{B}$ is nondegenerate [irreducible].

Remark 4.4. If $\mathscr{B}$ is right-resolving, then so are $\mathscr{G}_{1}$ and $\mathscr{G}_{2}$. If $\mathscr{B}$ is right-resolving and reduced, then so are $\mathscr{G}_{1}$ and $\mathscr{G}_{2}$.

A subshift $\Lambda$ is said to be bipartite if $A(\Lambda)$ is partitioned into two sets $C$ and $D$ such that for each $\left(\alpha_{i}\right)_{i \in Z} \in \Lambda$ and $i \in \mathbb{Z}, \alpha_{i}$ and $\alpha_{i+1}$ do not belong to the same set. The partition $C \cup D=A(\Lambda)$ is called the bipartite partition for the bipartite subshift $\Lambda$. (We also assume that the bipartite partition is specified when we mention a bipartite subshift.) For a bipartite subshift $\Lambda$ with bipartite partition $C \cup D=A(\Lambda)$, the two subshifts given by

$$
\left.\Lambda_{1}=\left\{\left(\alpha_{2 i-1} \alpha_{2 i}\right)\right)_{i \in \mathbf{Z}} \mid\left(\alpha_{i}\right)_{i \in \mathbf{Z}} \in \Lambda, \alpha_{0} \in D\right\}
$$

and

$$
\Lambda_{2}=\left\{\left(\alpha_{2 i-1} \alpha_{2 i}\right)_{i \in \mathbf{Z}} \mid\left(\alpha_{i}\right)_{i \in \mathbf{Z}} \in \Lambda, \alpha_{0} \in C\right\}
$$

are called the induced pair of subshifts of $\Lambda$.

A bipartite topological Markov chain and its induced pair of subshifts were used in $[4$, p. 99] to prove Williams' theorem.

THEOREM 4.5. Let $\Lambda$ be a bipartite sofic system. Let $\Lambda_{1}$ and $\Lambda_{2}$ be the induced pair of subshifts of $\Lambda$. Then $\mathscr{K}_{\Lambda}$ is a bipartite $\lambda$-graph, and $\mathscr{K}_{\Lambda_{1}}$ and $\mathscr{K}_{\Lambda_{2}}$ are the bipartite pair of $\lambda$-graphs of $\mathscr{K}_{\Lambda}$.

Proof. Let $C \cup D=A(\Lambda)$ be the bipartite partition for $\Lambda$. Let $\Lambda_{-}^{\mathrm{C}}=$ $\left\{\cdots \alpha_{-1} \alpha_{0} \in \Lambda_{-} \mid \alpha_{0} \in C\right\}$ and let $\Lambda_{-}^{D}$ be defined similarly. Then $\mathscr{K}_{\Lambda}$ is a bipartite $\lambda$-graph whose bipartite vertex-sets are $\left\{E_{\Lambda}(\delta) \mid \delta \in \Lambda_{-}^{D}\right\}$ and $\left\{E_{\Lambda}(\delta) \mid \delta \in \Lambda_{-}^{C}\right\}$. For each $\delta=\cdots \alpha_{-1} \alpha_{0}$ in $\Lambda_{-}^{D}$, let $p(\delta)$ denote the element $\cdots\left(\alpha_{-3} \alpha_{-2}\right)\left(\alpha_{-1} \alpha_{0}\right)$ in $\left(\Lambda_{1}\right)_{-}$. It is clear that for $\delta, \delta^{\prime} \in \Lambda_{-}^{D}, \delta E_{\Lambda} \delta^{\prime}$ iff $p(\delta) E_{\Lambda_{1}} p\left(\delta^{\prime}\right)$. Hence the correspondence between $E_{\Lambda}[\delta]$ and $E_{\Lambda_{\mathrm{t}}}[p(\delta)]$ is $1-1$. Let $\delta, \delta^{\prime} \in \Lambda_{-}^{D}$ and let $c d \in L(\Lambda)$ with $c \in C$ 
and $d \in D$. There exists a path of length 2 generating $c d$ and going from $E_{\Lambda}[\delta]$ to $E_{\Lambda}\left[\delta^{\prime}\right]$ in $\mathscr{K}_{\Lambda}$ iff there exists an arc labelled $c d$ going from $E_{\Lambda_{1}}[p(\delta)]$ to $E_{\Lambda_{1}}\left[p\left(\delta^{\prime}\right)\right]$ in $\mathscr{K}_{\Lambda_{1}}$, because $\delta c d E_{\Lambda} \delta^{\prime}$ iff $p(\delta)(c d) E_{\Lambda_{1}} p\left(\delta^{\prime}\right)$. Therefore $\mathscr{K}_{\Lambda_{1}}$ is one of the bipartite pair of $\lambda$-graphs of $\mathscr{K}_{\Lambda}$.

Similarly, $\mathscr{K}_{\Lambda_{2}}$ is the other one of the bipartite pair of $\lambda$-graphs of $\mathscr{K}_{\Lambda}$.

COROllary 4.6. Let $\phi: \Lambda \rightarrow \Omega$ be a bipartite code between sofic systems. Let $\theta: \Sigma \rightarrow \Lambda$ and $\pi: \Gamma \rightarrow \Omega$ be the right Krieger covers for $\Lambda$ and $\Omega$, respectively. Then the following statements are valid.

(1) There exists a bipartite Krieger $\lambda$-graph $\mathscr{B}$ such that $\mathscr{K}_{\Lambda}$ and $\mathscr{K}_{\Omega}$ are the bipartite pair of $\lambda$-graphs of $\mathscr{B}$, up to recoding of symbols.

(2) There exists a bipartite code $\eta: \Sigma \rightarrow \Gamma$ such that the diagram

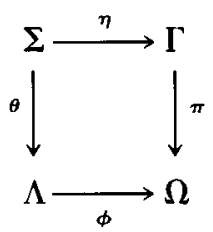

commutes.

(3) $M\left(\mathscr{K}_{\Lambda}\right)$ and $M\left(\mathscr{K}_{\Omega}\right)$ are strong shift equivalent in 1 step.

Proof. Let $f: A(\Lambda) \rightarrow C D$ be a bipartite expression inducing $\phi$. We may assume that $C \cap D=\varnothing$. Let $f_{1}: A(\Lambda) \rightarrow C$ and $f_{2}: A(\Lambda) \rightarrow D$ be such that

$$
f(a)=f_{1}(a) f_{2}(a), \quad a \in A(\Lambda) .
$$

For each $\alpha=\left(\alpha_{i}\right)_{i \in \mathbf{Z}} \in \Lambda$, let $q(\alpha)$ be the element of $(C \cup D)^{\mathbf{Z}}$ defined by $q(\alpha)=$ $\left(\delta_{i}\right)_{i \in Z}, \delta_{2 i-1}=f_{1}\left(\alpha_{i}\right)$ and $\delta_{2 i}=f_{2}\left(\alpha_{i}\right), i \in \mathbb{Z}$. Let $\Delta$ be the subshift given by

$$
\Delta=\{q(\alpha) \mid \alpha \in \Lambda\} \cup\{\sigma q(\alpha) \mid \alpha \in \Lambda\}
$$

where $\sigma$ is the shift map on $(C \cup D)^{Z}$. Then $\Delta$ is a bipartite sofic system, and $\Lambda$ and $\Omega$ are the induced pair of subshifts of $\Delta$, up to recoding of symbols. Hence, by theorem $4.5, \mathscr{K}_{\Lambda}$ and $\mathscr{K}_{\Omega}$ are the induced pair of $\lambda$-graphs of $\mathscr{K}_{\Delta}$, up to recoding of symbols. Thus (1) is proved, (2) follows from (1) and remark 4.2, and (3) follows from (1) and remark 4.1.

THEOREM 4.7. Let $\Lambda$ be a bipartite transitive sofic system. Let $\Lambda_{1}$ and $\Lambda_{2}$ be the induced pair of subshifts of $\Lambda$. Then $\mathscr{F}_{\Lambda}$ is a bipartite $\lambda$-graph, and $\mathscr{F}_{\Lambda_{1}}$ and $\mathscr{F}_{\Lambda_{2}}$ are the bipartite pair of $\lambda$-graphs of $\mathscr{F}_{\Lambda}$.

Proof. Let $C \cup D=A(\Lambda)$ be the bipartite partition for $\Lambda$. Since $\Lambda$ is a bipartite subshift, it is observed that $\mathscr{F}_{\Lambda}$ is a bipartite $\lambda$-graph with bipartite vertex-sets $U$ and $V$, where $U$ is the set of terminal vertices of the arcs labelled a symbol in $D$ and $V$ is the set of terminal vertices of the arcs labelled a symbol in $C$. Let $\mathscr{G}_{1}$ and $\mathscr{G}_{2}$ be the induced pair of $\lambda$-graphs of $\mathscr{F}_{\Lambda}$ having vertex-sets $U$ and $V$, respectively. Then, since $L\left(\mathscr{F}_{\Lambda}\right)=L(\Lambda)$, it follows that $L\left(\mathscr{G}_{1}\right)=L\left(\Lambda_{1}\right)$ and $L\left(\mathscr{G}_{2}\right)=L\left(\Lambda_{2}\right)$. Since $\mathscr{F}_{\Lambda}$ is a Fischer $\lambda$-graph (i.e. irreducible, right-resolving and reduced), so is each of $\mathscr{G}_{1}$ and $\mathscr{G}_{2}$ (remarks 4.3 and 4.4). Thus $\mathscr{G}_{1}=\mathscr{F}_{\Lambda_{1}}$ and $\mathscr{G}_{2}=\mathscr{F}_{\Lambda_{2}}$. 
By a proof similar to that of corollary 4.6 , we also have:

COROLlARY 4.8. Let $\phi: \Lambda \rightarrow \Omega$ be a bipartite code between transitive sofic systems. Let $\theta: \Sigma \rightarrow \Lambda$ and $\pi: \Gamma \rightarrow \Omega$ be the right Fischer covers for $\Lambda$ and $\Omega$, respectively. Then the following statements are valid.

(1) There exists a bipartite Fischer $\lambda$-graph $\mathscr{B}$ such that $\mathscr{F}_{\Lambda}$ and $\mathscr{F}_{\Omega}$ are the bipartite pair of $\lambda$-graphs of $\mathscr{B}$, up to recoding of symbols.

(2) There exists a bipartite code $\eta: \Sigma \rightarrow \Gamma$ such that the diagram

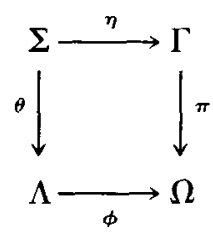

commutes.

(3) $M\left(\mathscr{F}_{\Lambda}\right)$ and $M\left(\mathscr{F}_{\Omega}\right)$ are strong shift equivalent in 1 step.

Proofs of theorems 3.3-3.6. Theorems 3.3 and 3.4 follow from corollaries 4.6 and 4.8 .

The 'only if' parts of theorems 3.5 and 3.6 follow from theorem 2.4 and corollaries 4.6 and 4.8.

Let $\theta: \Sigma \rightarrow \Lambda$ and $\pi: \Gamma \rightarrow \Omega$ be any two sofic covers. If the representation matrices of $\theta$ and $\pi$ are strong shift equivalent in 1 step, then there exists a bipartite $\lambda$-graph whose induced pair of $\lambda$-graphs are the $\lambda$-graph representing $\theta$ and that representing $\pi$, up to recoding of symbols (see remark 4.1), so that there exist bipartite codes $\eta_{1}: \Sigma \rightarrow \Gamma$ and $\phi_{1}: \Lambda \rightarrow \Omega$ such that $\pi \eta_{1}=\phi_{1} \theta$, by remark 4.2. Therefore if the representation matrices of $\theta$ and $\pi$ are strong shift equivalent, then there exist topological conjugacies $\eta: \Sigma \rightarrow \Gamma$ and $\phi: \Lambda \rightarrow \Omega$ such that $\pi \eta=\phi \theta$. Hence, we have proved more than the 'if' parts of theorems 3.5 and 3.6 (see $\S 6$ ).

Remark. There is a simple procedure which, given $M\left(\mathscr{K}_{\Lambda}\right)\left[M\left(\mathscr{F}_{\Lambda}\right)\right]$, where $\Lambda$ is any sofic system [transitive sofic system], and any bipartite expression $f$ of $A(\Lambda)$, calculates directly from them the representation matrix of the bipartite Krieger [Fischer] $\lambda$-graph whose induced pair of $\lambda$-graphs are $\mathscr{K}_{\Lambda}\left[\mathscr{F}_{\Lambda}\right]$ (up to recoding of symbols) and $\mathscr{K}_{\phi(\Lambda)}\left[\mathscr{F}_{\phi(\Lambda)}\right]$, and hence $M\left(\mathscr{K}_{\phi(\Lambda)}\right)\left[M\left(\mathscr{F}_{\phi(\Lambda)}\right)\right]$, where $\phi$ is the standard bipartite code induced by $f$.

\section{Automata and canonical sofic covers}

In this section, we give a method based on finite automata theory to construct Krieger covers for a given sofic system. A similar method to construct Fischer covers for a given transitive sofic system, was presented in [11].

A right resolving $\lambda$-graph is called an automaton if one of its vertices is specified as the starting vertex. For an automaton $\mathscr{A}$ with starting vertex $u_{0}, L_{u_{0}}(\mathscr{A})$ is called the language accepted by $\mathscr{A}$. $\mathscr{A}$ is said to be minimal if $\mathscr{A}$ is reduced and for each vertex $u \neq u_{0}$, there exists a path going from $u_{0}$ to $u$ in $\mathscr{A}$. It is well known in finite automata theory and will also become clear in the following exposition that $\mathscr{A}$ is minimal iff it is an automaton with the least number of vertices that accepts $L_{u_{0}}(\mathscr{A})$. 
Two automata $\mathscr{A}_{1}$ and $\mathscr{A}_{2}$ are said to be isomorphic if there exists an isomorphism $(g, h)$ of $\mathscr{A}_{1}$ onto $\mathscr{A}_{2}$ (of $\lambda$-graphs) such that $g$ maps the starting vertex of $\mathscr{A}_{1}$ to that of $\mathscr{A}_{2}$. ( $\mathrm{g}$ is called an isomorphism of automata.) In what follows, isomorphic automata will be identified.

Finite automata theory also shows that for any sofic system $\Lambda$, there exists a unique minimal automaton that accepts $L(\Lambda)$. We give a direct proof for its existence and uniqueness for completeness and also give a standard method in finite automata theory to construct it from any given $\lambda$-graph $\mathscr{G}$ with $L(\mathscr{G})=L(\Lambda)$ (i.e. from any given cover for $\Lambda$ ).

(1) First we give a minimal automaton $\mathscr{A}_{\Lambda}$ accepting $L(\Lambda)$, for any sofic system $\Lambda$. Put $A=A(\Lambda)$ and put $L=L(\Lambda)$. Let $\hat{L}=L \cup\{\varepsilon\}$, where $\varepsilon$ is the unit element of the monoid $A^{*}$. Let

$$
L_{x}=\{y \in L \mid x y \in L\}
$$

for each $x \in \hat{L}$. As will be proved below, $\left\{L_{x} \mid x \in \hat{L}\right\}$ is finite. The vertex-set of $\mathscr{A}_{\Lambda}$ is $\left\{L_{x} \mid x \in \hat{L}\right\}$ and the starting vertex of $\mathscr{A}_{\Lambda}$ is $L_{\varepsilon}=L$. For each vertex $L_{x}, x \in \hat{L}$ and $a \in A$, a unique arc labelled $a$ goes from $L_{x}$ to $L_{x a}$ if $x a \in L$. It is easy to see that $\mathscr{A}_{\Lambda}$ is minimal and accepts $L(\Lambda)$.

(2) (Subset-construction). We show that $\left\{L_{x} \mid x \in \hat{L}\right\}$ is finite and at the same time, give a standard method (subset-construction) to construct an automaton accepting $L(\Lambda)$ from any $\lambda$-graph $\mathscr{G}$ such that $L(\mathscr{G})=L(\Lambda)$ (i.e. a cover for $\Lambda$ ). Let $U$ be the vertex set of $\mathscr{G}$. For any $V \subset U$ and $x \in A^{*}$, define $S(V, x)$ to be the set of all terminal vertices of the paths going from some vertex in $V$ and generating $x$. (We define $S(V, \varepsilon)=V$.) We can define an automaton $\mathscr{A}_{\mathscr{G}}^{S}$ as follows. The vertex-set of $\mathscr{A}_{\mathscr{G}}^{S}$ is $\left\{S(U, x) \mid x \in A^{*}, S(U, x) \neq \varnothing\right\}$. The starting vertex of $\mathscr{A}_{\mathscr{G}}^{S}$ is $U$. For each vertex $S(U, x) \neq \varnothing, x \in A^{*}$, and $a \in A(\mathscr{G})=A$, a unique arc labelled $a$ goes from $S(U, x)$ to $S(S(U, x), a)=S(U, x a)$ if $S(U, x a) \neq \varnothing$. Since $x \in \hat{L}$ iff $S(U, x) \neq \varnothing, \mathscr{A}_{\mathscr{G}}^{S}$ accepts $L=L(\mathscr{G})$. Clearly, we have

$$
L_{S(U, x)}\left(\mathscr{A}_{\mathscr{G}}^{S}\right)=L_{x}
$$

for each $x \in \hat{L}$. Since $\{S(U, x) \mid x \in \hat{L}\}$ is finite, so is $\left\{L_{x} \mid x \in \hat{L}\right\}$.

(3) (Uniqueness). Let $\mathscr{A}$ be any automaton accepting $L(\Lambda)$ such that for each vertex $v$ of $\mathscr{A}$ which is not the starting vertex, there exists a path going from the starting vertex to $v$. Let $u_{0}$ be the starting vertex of $\mathscr{A}$. For each $x \in \hat{L}$, let $s\left(u_{0}, x\right)$ denote the terminal vertex of the path going from $u_{0}$ and generating $x$ (we define $\left.s\left(u_{0}, \varepsilon\right)=u_{0}\right)$. Then $L_{s\left(u_{0}, x\right)}(\mathscr{A})=L_{x}$ for all $x \in \hat{L}$. Therefore if $\mathscr{A}$ is minimal, the mapping of the vertex-set of $\mathscr{A}$ into $\left\{L_{x} \mid x \in \hat{L}\right\}$ that maps $s\left(u_{0}, x\right)$ to $L_{x}$, is one-to-one and onto and hence an isomorphism (of automata) of $\mathscr{A}$ onto $\mathscr{A}_{\Lambda}$.

(4) (Minimization). To obtain $\mathscr{A}_{\Lambda}$ from $\mathscr{A}$, let $\left\{C_{i}\right\}$ be the equivalence classes of the equivalence relation of vertices of $\mathscr{A}$. Let $\mathscr{A}^{\prime}$ be the automaton defined as follows: the vertex set of $\mathscr{A}^{\prime}$ is $\left\{C_{i}\right\}$ and the starting vertex of $\mathscr{A}^{\prime}$ is the class $C_{0}$ that contains $u_{0}$; for equivalence classes $C_{i}$ and $C_{j}$ and $a \in A$, a unique arc labelled $a$ goes from $C_{i}$ to $C_{j}$ if there exists an arc labelled $a$ going from a vertex in $C_{i}$ to that in $C_{j}$ in $\mathscr{A}$. Clearly $\mathscr{A}^{\prime}$ is equal to $\mathscr{A}_{\Lambda}$. This process is called the minimization of $\mathscr{A}$. We note 
that a finite procedure to obtain the equivalence classes $\left\{C_{i}\right\}$ is well known in finite automata theory.

Let $\mathscr{A}$ be an automaton over an alphabet $A$ with starting vertex $u_{0}$. For each vertex $u$ of $\mathscr{A}$, let $L_{u_{0}}^{u}(\mathscr{A})$ denote the set of words generated by the paths going from $u_{0}$ to $u$. A vertex $v$ of $\mathscr{A}$ is called a $K$-vertex of $\mathscr{A}$ if $L_{u_{0}}^{v}(\mathscr{A})$ contains infinitely many terminal subwords of some left infinite sequence over $A$. (For a left infinite sequence $\gamma=\cdots \gamma_{-1} \gamma_{0}, \gamma_{-i} \gamma_{-i+1} \cdots \gamma_{0}$ is a terminal subword of $\gamma$ for each $i \geq 0$.)

Proposition 5.1. Let $\Lambda$ be a sofic system. Then $\mathscr{K}_{\Lambda}$ is equal to the maximal sub- $\lambda$-graph of $\mathscr{A}_{\Lambda}$ whose vertex-set consists of all the $K$-vertices of $\mathscr{A}_{\Lambda}$.

Proof. Let $\gamma=\cdots \gamma_{-1} \gamma_{0} \in \Lambda_{-}$. For each $i \geq 0$, let $s_{i}$ be the terminal vertex of the path in $\mathscr{A}_{\Lambda}$ going from the starting vertex $u_{0}$ of $\mathscr{A}_{\Lambda}$ and generating $\gamma_{-i} \gamma_{-i+1} \cdots \gamma_{0}$. Since the number of vertices of $\mathscr{A}_{\Lambda}$ is finite, there exists a vertex $s(\gamma)$ such that $s_{i}=s(\gamma)$ for infinitely many $i \geq 0$. Since

$$
L_{s_{i}}\left(\mathscr{A}_{\Lambda}\right) \supset L_{s_{i+1}}\left(\mathscr{A}_{\Lambda}\right) \quad \text { for all } i \in \mathbb{N},
$$

it follows that if $s_{j}=s(\gamma)$ for some $j \geq 0$, then

$$
L_{s_{i}}\left(\mathscr{A}_{\Lambda}\right)=L_{s(\gamma)}\left(\mathscr{A}_{\Lambda}\right) \quad \text { for all } i \geq j,
$$

which implies that $s_{i}=s(\gamma)$ for all $i \geq j$, because $\mathscr{A}_{\Lambda}$ is reduced. Thus $s(\gamma)$ is unique for $\gamma$. Clearly $s(\gamma)$ is a $K$-vertex of $\mathscr{A}_{\Lambda}$. Moreover, by (5.1) we have

$$
L_{s(\gamma)}\left(\mathscr{A}_{\Lambda}\right)=\left\{x \in A A^{*} \mid \gamma x \in \Lambda_{-}\right\} .
$$

If $v$ is a $K$-vertex of $\mathscr{A}_{\Lambda}$, then there exists a left infinite sequence $\delta$ such that $L_{u_{0}}^{v}\left(\mathscr{A}_{\Lambda}\right)$ contains infinitely many terminal subwords of $\delta$. Clearly $\delta \in \Lambda_{-}$and $v=s(\delta)$.

Thus a vertex $v$ of $\mathscr{A}(\Lambda)$ is a $K$-vertex iff $v=s(\gamma)$ for some $\gamma \in \Lambda_{-}$. By (5.2) and the reducedness of $\mathscr{A}_{\Lambda}$ we see that the correspondence between $E_{\Lambda}[\gamma]$ and $s(\gamma)$, where $\gamma \in \Lambda_{-}$, is $1-1$. It is easily seen that this correspondence gives an isomorphism between $\mathscr{K}_{\Lambda}$ and the maximal sub- $\lambda$-graph of $\mathscr{A}_{\Lambda}$ whose vertex-set is $\left\{s(\gamma) \mid \gamma \in \Lambda_{-}\right\}$.

If $\Lambda$ is a transitive sofic system, $\mathscr{F}_{\Lambda}$ is obtained as the unique ergodic component of $\mathscr{A}_{\Lambda}[11]$.

Example. Let $\Lambda$ be a transitive sofic system over $A=\{a, b, c, d, e\}$ such that

$$
M\left(\mathscr{F}_{\Lambda}\right)=\left(\begin{array}{lll}
a & b & c \\
d & a & 0 \\
e & 0 & a
\end{array}\right) .
$$

By subset construction, we have

$$
M\left(\mathscr{A}_{\Lambda}\right)=M\left(\mathscr{K}_{\Lambda}\right)=\left(\begin{array}{cccc}
a & d+e & b & c \\
0 & a & b & c \\
0 & d & a & 0 \\
0 & e & 0 & a
\end{array}\right) .
$$


Let $\Omega$ be a transitive sofic system over $B=\{a, b, \ldots, j\}$ such that

$$
M\left(\mathscr{F}_{\Omega}\right)=\begin{aligned}
& 1 \\
& 2 \\
& 3 \\
& 4 \\
& 5
\end{aligned}\left(\begin{array}{lllll}
0 & 0 & 0 & b & 0 \\
0 & 0 & 0 & 0 & b \\
c & d & a & 0 & 0 \\
e & f & g & a & 0 \\
h & i & j & 0 & a
\end{array}\right) .
$$

By subset construction we obtain $\mathscr{A}_{\Omega}$ such that $M\left(\mathscr{A}_{\Omega}\right)$ is given as

$$
\left.\begin{array}{c}
(1,2,3,4,5) \\
(4,5) \\
(3,4,5) \\
1 \\
2 \\
3 \\
4
\end{array} \quad \begin{array}{cccccccc}
0 & b & a & c+e+h & d+f+i & g+j & 0 & 0 \\
0 & a & 0 & e+h & f+i & g+j & 0 & 0 \\
5 & 0 & a & c+e+h & d+f+i & g+j & 0 & 0 \\
0 & 0 & 0 & 0 & 0 & 0 & b & 0 \\
0 & 0 & 0 & 0 & 0 & 0 & 0 & b \\
0 & 0 & 0 & c & d & a & 0 & 0 \\
0 & 0 & 0 & e & f & g & a & 0 \\
0 & 0 & 0 & h & i & j & 0 & a
\end{array}\right) .
$$

It is easy to see that

$$
M\left(\mathscr{K}_{\Omega}\right)=\left(\begin{array}{cccccc}
a & c+e+h & d+f+i & g+j & 0 & 0 \\
0 & 0 & 0 & 0 & b & 0 \\
0 & 0 & 0 & 0 & 0 & b \\
0 & c & d & a & 0 & 0 \\
0 & e & f & g & a & 0 \\
0 & h & i & j & 0 & a
\end{array}\right) .
$$

Note that $\mathscr{K}_{\Omega}$ is not the maximal non-degenerate sub- $\lambda$-graph of $\mathscr{A}_{\Omega}$. Let

$$
P=\left(\begin{array}{llllll}
a & d & c & 0 & 0 & 0 \\
0 & d & c & a & 0 & 0 \\
0 & 0 & 0 & 0 & a & 0 \\
0 & 0 & 0 & 0 & 0 & a
\end{array}\right) \text { and } Q=\left(\begin{array}{ccccc}
a & c+d & 0 & 0 \\
0 & 0 & b & 0 \\
0 & 0 & 0 & b \\
0 & a & 0 & 0 \\
0 & c & a & 0 \\
0 & d & 0 & a
\end{array}\right)
$$

Then we can see that $M\left(\mathscr{K}_{\Lambda}\right) \simeq P Q$ and $M\left(\mathscr{K}_{\Omega}\right) \simeq Q P$.

This example shows that an analogue of Krieger's theorems does not hold for the sofic covers which are represented by the maximal non-degenerate sub- $\lambda$-graphs of minimal automata.

\section{Concluding remarks}

In [2], topological conjugacy for sofic covers has been defined: two sofic covers $\theta: \Sigma \rightarrow \Lambda$ and $\pi: \Gamma \rightarrow \Omega$ are topologically conjugate if there exist topological conjugacies $\eta: \Sigma \rightarrow \Gamma$ and $\phi: \Lambda \rightarrow \Omega$ such that the diagram (3.1) commutes. As was proved in $\S 4$, if the representation matrices of two sofic covers are strong shift equivalent, then the sofic covers are topologically conjugate. The question of whether the converse 
is valid or not, naturally arises. The answer is affirmative: one can prove that if two sofic covers are topologically conjugate, then their representation matrices are strong shift equivalent. Thus Williams' theorem holds for sofic covers.

Recently, for the above claimed result, Toshihiro Hamachi has given a proof (based on the proof of theorem 2.4) which is simpler than my original one and can prove more. Hence a thorough treatment of the above subject is contained in [5].

I am indebted to the referee for his constructive criticism and useful suggestions which led to several great improvements in the organization and presentation of the paper. I should like to thank Mike Boyle for his valuable comments.

\section{REFERENCES}

[1] R. L. Adler \& B. Marcus. Topological entropy and equivalence of dynamical systems. Memoirs Amer. Math. Soc. 219 (1979).

[2] M. Boyle, B. Kitchens \& B. Marcus. A note on minimal covers for sofic systems. Proc. Amer. Math. Soc. 95 (1985), 403-411.

[3] R. Fischer. Graphs and symbolic dynamics. In Colloq. Math. Soc. János Bolyai 16, Topics in Information Theory. Keszthely, Hungary, 1975.

[4] J. Franks. Homology and Dynamical Systems. C.B.M.S. Regional Conference Series in Math. No. 49. Amer. Math. Soc.: Providence, R.I., 1982.

[5] T. Hamachi \& M. Nasu. Topological conjugacy for 1-block factor maps of subshifts and sofic covers. Preprint.

[6] M. A. Harrison. Introduction to Switching and Automata Theory. McGraw-Hill: New York, 1965.

[7] G. A. Hedlund. Endomorphisms and automorphisms of the shift dynamical system. Math. Systems Theory 3 (1969), 320-375.

[8] B. Kitchens \& S. Tuncel. Semi-groups and graphs for sofic systems. Israel J. Math. To appear.

[9] W. Krieger. On sofic systems, I. Israel J. Math. 48 (1984), 305-330.

[10] W. Krieger. On sofic systems, II. Preprint, Institut für Angewandte Mathematik der Universität Heidelberg.

[11] M. Nasu. An invariant for bounded-to-one factor maps between transitive sofic subshifts. Ergod. Th. \& Dynam. Sys. 5 (1985), 89-105.

[12] B. Weiss. Subshifts of finite type and sofic systems. Monatsh. Math. 77 (1973), 462-474.

[13] R. F. Williams. Classification of subshifts of finite type. Ann. of Math. 98 (1973), 120-153; Errata: Ann. of Math. 99 (1974), 380-381. 